\title{
Entrenamiento en Salvamento Deportivo. Estudio en la comunidad Gallega Coaching in Life Saving. Study in Galicia
}

\author{
J. Arturo Abraldes Valeiras, Nuria Rodríguez Suárez \\ Universidad Católica San Antonio de Murcia (España)
}

\begin{abstract}
Resumen: En el presente documento se analizan las características de los entrenamientos de los deportistas de salvamento acuático, tales como los recursos humanos, materiales, condiciones de entrenamiento y control del mismo, así como la práctica de otro tipo de deportes. La muestra del estudio está referida a los deportistas de Salvamento Acuático Deportivo que participaron en el Campeonato Gallego de Aguas Cerradas en el año 2006. Dicha muestra asciende a un total de 73 deportistas, de los cuales el 43,84\% (32) son mujeres y el 56,16\% (41) son varones. El análisis estadístico de los datos se realizó a través del paquete informático SPSS 12.0 en el entorno Windows XP Profesional, llevándose a cabo un análisis descriptivo de los datos (frecuencias absolutas, medias, porcentajes, desviaciones,...). Entre los resultados más destacados podemos apreciar una formación adecuada de los entrenadores, así como una valoración aceptable en relación a las condiciones de sus entrenamientos (piscina, gimnasio, material específico y pulsímetros). Una mayor presencia de entrenamientos estratégicos y tácticos frente a otro tipo de entrenamientos. Entrenamientos en grupo con una frecuencia de entrenamiento físico casi diaria en sesiones de hora y media. Por otro lado, se encuentra un bajo porcentaje de simultaneidad con otras prácticas deportivas y una menor dedicación a éstas, con respecto al salvamento acuático deportivo
\end{abstract}

Palabras clave: Entrenamiento. Factores de formación. Salvamento Acuático Deportivo. Volumen.

Abstract: TIn the present document are analyzed the characteristics of training of aquatic rescue sportspeople, as human resources, materials, training conditions and its control, as the practice of other kind of sports. The sample of the study is refered to sportspeople of Aquatic Sport Rescue that took part in the Campeonato Gallego de Aguas Cerradas in the year 2006. That sample rise to a total of 73 sportspeople, of which $43.84 \%$ (32) are women and 56.16\% (41) are men. The statistical analysis of data was carried out through the software package SPSS 12.0 in the Windows XP Professional environment, and a descriptive analysis of data (absolute frequencies, averages, percentages, deviations...) was carried out. Among the most highlighted results can be seen a adequate training, as well as an acceptable assessment according to the conditions of coaching (swimming pool, gymnasium, specific material and pulsometers).

Key words: Coaching. Training factors. Lifesaving. Volume.

\section{Introducción}

El salvamento acuático es una modalidad deportiva que en España tiene pocos adeptos, y que en comparación con el número de licencias deportivas que presentan otras modalidades, éste está muy por debajo de lo que deseamos. Sin embargo, es un deporte practicado en España y que presenta unos grandes resultados a nivel nacional e internacional.

Como deporte minoritario que es, nos encontramos que hay pocos estudios de rigor sobre el mismo, y que por tanto, no se somete al análisis ni a la crítica de los investigadores, que ayudan a buscar nuevas técnicas y métodos para que éste evolucione y sea más competitivo y mejor.

Generalmente la evolución en este deporte se basa en el ensayoerror, así como en la observación de las técnicas y sistemas de entrenamiento que hacen otros deportistas, clubes o entrenadores. Son estos últimos los que dan una verdadera calidad al entrenamiento de los deportistas, entendiendo que a mayor formación, tanto general como específica del deporte, los entrenadores sabrán establecer los mejores planteamientos y sacar el máximo provecho del deportista.

Es por ello que nos embarga la duda de conocer como son los entrenadores que están al cargo de los deportistas que practican este deporte, así como ver las características que tienen sus entrenamientos en cuanto a las instalaciones y materiales utilizados. El tipo de entrenamiento que proponen a sus deportistas y la evolución y control de los avances en sus resultados. Por ello nos hemos planteado este estudio, intentando dar respuesta a los entrenamientos que presentan los entrenadores de los mejores deportistas a nivel autonómico en Galicia.

Fundamentalmente, en los primeros pasos de la iniciación deportiva, entendemos que se debe buscar una formación integral del deportista, al igual que a lo largo de toda su carrera. Aunque a medida que se avanza en edad, formación y experiencia, la prioridad en el deporte se va centrando, cada vez más, en la obtención de resultados deportivos. Este

Fecha recepción: 10-06-07 - Fecha envío revisores: 10-06-07 - Fecha de aceptación: 05-08-07 Correspondencia: Arturo Abraldes Valeiras

Campus de los Jerónimos, s/n. 30107

GUADALUPE (Murcia) ESPAÑA

E-mail: AAbraldes@pdi.ucam.edu aspecto, creemos que está muy relacionado con la formación que presenten los entrenadores y/o formadores de los deportistas, así como las condiciones de entrenamiento que tienen que «soportar» los deportistas.

Como punto de partida para el análisis de este deporte, este trabajo puede ser interesante ya que nos muestra la realidad existente y, una vez conocida, se pueden aplicar medidas para corregir su evolución o modificarla en dirección más adecuada.

\section{Método}

La población de estudio comprende a los deportistas de SalvamentoAcuático Deportivo de Galicia, con licencia federativa en el año 2006. La muestra queda definida por deportistas de Salvamento Acuático Deportivo que participan en el Campeonato Gallego de Aguas Cerradas en el año 2006. Dicha muestra asciende a un total de 73 deportistas, de los cuales el 43,84\% (32) son mujeres y el 56,16\% (41) son varones.

Previo al Campeonato Gallego y con antelación suficiente elaboró un cuestionario específico para el presente estudio. El mismo día en el que se iniciaba la competición, se realizó una pequeña entrevista con el Director de Competición del Campeonato donde se le explicó los obje-

\begin{tabular}{|c|c|c|c|c|c|c|c|c|c|c|}
\hline \multicolumn{11}{|c|}{ Tabla 1. Características de la muestra. Datos expresados en medias. } \\
\hline Género & & Casos & \multicolumn{2}{|c|}{ Edad } & \multicolumn{2}{|c|}{ Peso } & \multicolumn{2}{|c|}{ Altura } & \multicolumn{2}{|c|}{ Masa Corporal } \\
\hline \multirow{2}{*}{\multicolumn{2}{|c|}{ Femenino }} & $\mathrm{x}$ & \multicolumn{2}{|c|}{16,58} & \multicolumn{2}{|c|}{59,13} & \multicolumn{2}{|c|}{162,13} & \multicolumn{2}{|c|}{22,49} \\
\hline & & sd & \multicolumn{2}{|c|}{ $\pm 2,41$} & \multicolumn{2}{|c|}{ $\pm 6,87$} & \multicolumn{2}{|c|}{ $\pm 6,40$} & \multicolumn{2}{|c|}{ $\pm 2,22$} \\
\hline \multirow{2}{*}{ Masculino } & & $\mathrm{x}$ & \multicolumn{2}{|c|}{19,78} & \multicolumn{2}{|c|}{72,02} & \multicolumn{2}{|c|}{175,96} & \multicolumn{2}{|c|}{23,25} \\
\hline & & sd & \multicolumn{2}{|c|}{ $\pm 5,49$} & \multicolumn{2}{|c|}{ $\pm 10,67$} & \multicolumn{2}{|c|}{ $\pm 7,67$} & \multicolumn{2}{|c|}{ $\pm 2,40$} \\
\hline \multirow{2}{*}{ Total } & & $\mathrm{x}$ & \multicolumn{2}{|c|}{18,38} & \multicolumn{2}{|c|}{63,37} & \multicolumn{2}{|c|}{169,74} & \multicolumn{2}{|c|}{22,92} \\
\hline & & $\mathrm{sd}$ & \multicolumn{2}{|c|}{ $\pm 4,67$} & \multicolumn{2}{|c|}{ $\pm 11,18$} & \multicolumn{2}{|c|}{ $\pm 9,81$} & \multicolumn{2}{|c|}{ $\pm 2,35$} \\
\hline \multicolumn{11}{|c|}{$\begin{array}{c}\text { Ley enda: }(n) \text { nimero de casos, (media) datos en términos medios, (sd) desviación tipica, (Masa Corporal) el indice } \\
\text { resulta de obtener el coeficiente entre el peso del deportista dividido por la altura al cuadrado. }\end{array}$} \\
\hline \multicolumn{11}{|c|}{ Tabla 2. Titulación del entrenador en la actualidad $(\mathrm{n}=72)$} \\
\hline Género & C & M.N. & A.N & S.N & M.S. & A.S. & S.S. & Dipl. & Ledo. & $\mathrm{N} / \mathrm{C}$ \\
\hline \multirow{2}{*}{ Femenino } & $\mathrm{n}$ & 0 & 3 & 0 & 1 & 2 & 2 & 1 & 3 & 6 \\
\hline & $\%$ & $0 \%$ & $10,34 \%$ & $0,00 \%$ & $3,45 \%$ & $6,90 \%$ & $6,90 \%$ & $3,45 \%$ & $10,34 \%$ & $20,69 \%$ \\
\hline Proulin & $\mathrm{n}$ & 0 & 8 & 1 & 4 & 2 & 3 & 6 & 7 & 11 \\
\hline Mascunino & $\%$ & $0 \%$ & $16,33 \%$ & $2,04 \%$ & $8,16 \%$ & $4,08 \%$ & $6,12 \%$ & $12,24 \%$ & $14,29 \%$ & $22,45 \%$ \\
\hline Total & $\mathrm{n}$ & 0 & 22 & 2 & 5 & 4 & 5 & 7 & 10 & 17 \\
\hline Iotal & $\%$ & $0, \%$ & $24,44 \%$ & $2,22 \%$ & $5,56 \%$ & $4,44 \%$ & $5,56 \%$ & $7,78 \%$ & $11,11 \%$ & \begin{tabular}{|l|l|}
$18,89 \%$ \\
\end{tabular} \\
\hline
\end{tabular}


tivos y el procedimiento que se pretendía seguir para llevar a cabo la investigación, así como la posibilidad de realizar la investigación sin mermar el desarrollo de la competición.

La toma de datos se realizó entre los días 04 y 05 de marzo de 2006, fechas en las que se realizaba el Campeonato Gallego de Aguas Cerradas. La administración del cuestionario se realizó en un aula anexa a la piscina de competición, dispuesta en los laterales de la misma, en ella se encontraban suficientes sillas y mesa para facilitar la escritura.

El procedimiento a la hora de la toma de datos se realizaba de la siguiente forma. Una vez sentados los deportistas en el lugar pertinente, se realizaba una presentación formal, donde se indicaba el nombre del investigador, los objetivos que se pretendían con la investigación. Una vez finalizada la presentación, se distribuían los cuestionarios a los deportistas, que procedían a contestarlos. Durante el desarrollo de la toma de datos, los deportistas podían realizar las preguntas pertinentes para solucionar sus dudas.

La introducción de datos se realizó a través de una plantilla diseñada, para tal fin, con el programa Excel del paquete informático Microsoft Office 2003 del entorno Windows XP Profesional, codificando y categorizando cada una de las variables. Una vez finalizada la introducción de los datos se procedió a la depuración de los mismos. Ésta se realizó principalmente recodificando variables y generando nuevas por la combinación de otras.

El principal material para la toma de datos fue un cuestionario elaborado para la ocasión, el cual contenía un total de 63 preguntas específicas (20 abiertas y 43 cerradas). A su vez, las preguntas cerradas disponían de, según los casos, otras preguntas afines al contenido de la pregunta principal o una opción («otros») para aclarar o completar la respuesta.

El cuestionario diseñado como herramienta para la recogida de información pasó por diferentes fases de elaboración, así como por distintos filtros de validación y fiabilidad. La elección del cuestionario como instrumento atiende a ventajas que defienden autores como Gómez (1990), Fernández Balboa (1997) y que fundamentalmente se centran en recoger información a grandes muestras.

Confeccionada la primera versión del cuestionario se inició el proceso de validación del mismo. Este proceso consistió en tres grandes apartados: una prueba piloto, validación por consenso de expertos y validación por fiabilidad.

El análisis estadístico de los datos se realizó con el paquete informático SPSS 12.0 en el entorno Windows XP Profesional, llevándose a cabo un análisis descriptivo de los datos (frecuencias absolutas, medias, porcentajes, desviaciones, ...).

\section{Resultados y discusión}

\subsection{En relación a los recursos humanos}

Entre los resultados hallados nos encontramos que, analizando los recursos humanos que los deportistas tienen a su servicio, el común a todos es la figura del entrenador. En este apartado, los deportistas

\begin{tabular}{|c|c|c|c|c|c|c|}
\hline Género & Casos & Bipn & Dogular & Yor & No hay & Impres. \\
\hline \multirow{2}{*}{ Femenino } & $\mathrm{n}$ & 27 & 3 & 1 & 0 & 29 \\
\hline & $\%$ & $87,10 \%$ & $9,68 \%$ & $3,23 \%$ & $0,00 \%$ & $90,63 \%$ \\
\hline \multirow{2}{*}{ Masculino } & $\mathrm{n}$ & 30 & 3 & 0 & 1 & 38 \\
\hline & $\%$ & $88,24 \%$ & $8,82 \%$ & $0,00 \%$ & $2,94 \%$ & $97,44 \%$ \\
\hline \multirow{2}{*}{ Total } & $\mathrm{n}$ & 57 & 6 & 1 & 1 & 67 \\
\hline & $\%$ & $87,69 \%$ & $9,23 \%$ & $1,54 \%$ & $1,54 \%$ & $94,37 \%$ \\
\hline \multicolumn{7}{|c|}{$\begin{array}{l}\text { Leyenda: (Bien) valoración de bien, (Regular) valoración de regular, (Mal) valoración de mal, (No hay) no tengo } \\
\text { entrenador, (Impres.) es imprescindible para los entrenamientos. }\end{array}$} \\
\hline Género & Casos & Bien & Regular & Mal & No hay & Impres. \\
\hline \multirow{2}{*}{ Femenino } & $\mathrm{n}$ & 17 & 12 & 0 & 1 & 20 \\
\hline & $\%$ & $56,67 \%$ & $40,00 \%$ & $0,00 \%$ & $3,33 \%$ & $64,52 \%$ \\
\hline \multirow{2}{*}{ Masculino } & $\mathrm{n}$ & 19 & 11 & 2 & 4 & 27 \\
\hline & $\%$ & $52,78 \%$ & $30,56 \%$ & $5,56 \%$ & $11,11 \%$ & $75,00 \%$ \\
\hline \multirow{2}{*}{ Total } & $\mathrm{n}$ & 36 & 23 & 2 & 5 & 47 \\
\hline & $\%$ & $54,55 \%$ & $34,85 \%$ & $3,03 \%$ & $7,58 \%$ & $70,15 \%$ \\
\hline
\end{tabular}

manifiestan haber tenido una media superior a tres entrenadores a lo largo de su vida deportiva $(3,37 \pm 1,63)$.

El mayor porcentaje de titulación encontrada en los entrenadores (Tabla 2) que actualmente tienen los deportistas es la de entrenador auxiliar de natación (24,44\%), seguida de la de licenciado en Educación Física $(11,11 \%)$, monitor de salvamento y entrenador superior de salvamento $(5,56 \%)$. Son destacables estos datos ya que la titulación más habitual no es específica de esta disciplina, sino de otra afin como es la natación. Cierto es que hace años que la Federación de Salvamento y Socorrismo de Galicia (FESSGA) no realiza cursos de formación para entrenadores, encontrándonos por tanto, datos que justifican este bajo porcentaje de profesionales con dicha formación.

Si atendemos a los diferentes grupos de titulación nos encontramos que el 5,56\% son monitores, el 28,88\% entrenadores auxiliares y el $7,78 \%$ entrenadores superiores. Datos que nos vienen a indicar la especificad en el campo de entrenamiento por parte de los entrenadores que dirigen los diferentes clubes gallegos.

Si analizamos la formación de los entrenadores actuales en función del grado de especificad, nos encontramos que el $32,22 \%$ tiene formación en el ámbito de la natación y el 24,45\% en el salvamento acuático deportivo. Resultados que nos indican que los entrenadores gallegos tienen una formación más específica en el mundo de la natación. Sibien, esto es lógico dada la ausencia de formación como comentábamos anteriormente.

Los propios deportistas hacen una valoración de sus entrenadores, y el 59,68\% de ellos consideran buena su intervención en su entrenamiento. Además, su trabajo se reconoce como imprescindible para su evolución deportiva en el 79,37\% de los casos.

\subsection{En relación a los recursos materiales}

En relación con los datos obtenidos al analizar los recursos materiales, hemos preguntado por la valoración de las instalaciones más usuales (piscina, gimnasio) y materiales más utilizados en un entrenamiento de calidad (material específico de salvamento, pulsímetros). Para completar dicha información, se solicitó que nos indicasen, si esa instalación o material era imprescindible para su formación como deportista de esta disciplina.

En el presente estudio, el $87,69 \%$ de los deportistas manifiestan que las piscinas en las que entrenan tienen unas condiciones buenas y aceptables. Mientras que el 10,77\% manifiesta que ésta está regular o mal. En el 1,54\% de los casos indican que no tienen piscina donde entrenar(Tabla3). Lo que en principio podría resultar alarmante, ya que éste es un deporte acuático, no lo es tanto, si interpretamos que este porcentaje puede ser debido a deportistas especialistas de alguna modalidad de aguas abiertas, como las de correr en la arena o las específicas de tablas de rescate o ski.

La utilización de la piscina para un buen entrenamiento de los deportistas en este deporte es resaltada por el $94,37 \%$ de ellos, entendiéndose como una instalación básica y fundamental para su desarrollo como deportista.

En relación al gimnasio como instalación de entrenamiento tenemos que decir que, para los deportistas no resulta tan imprescindible (70,15\%) como lo era la piscina. Dato que se refuerza con el resultado de que el $7,58 \%$ manifiesta no tener esta instalación para entrenar. De entre los deportistas que utilizan esta instalación deportiva, sus porcentajes son parecidos en los criterios de bien y regular, ocupando un $89,40 \%$ de los casos.

El salvamento y socorrismo se diferencia, entre otras muchas cosas, de la natación por la utilización de un material específico en sus pruebas (tubo de rescate, aletas, tabla, ski). Los recursos materiales en salvamento acuático deportivo son muy específicos y costosos. Es por ello que una buena dotación de material deportivo específico otorga una buena calidad de entrenamiento. Esta afirmación se justifica con la opinión de los deportistas que indican en un $80,00 \%$ que los materiales específicos son imprescindibles para llevar a cabo un buen entrenamiento y conseguir buenos resultados deportivos. 


\begin{tabular}{|c|c|c|c|c|c|c|}
\hline \multicolumn{7}{|c|}{ Tabla 5. Valoración del material específico para entrenar $(n=64)$. Imprescindible $(n=65)$} \\
\hline Género & Casos & Bien & Regular & Mal & No hay & Impres. \\
\hline \multirow{2}{*}{ Femenino } & $\mathrm{n}$ & 19 & 8 & 0 & 3 & 24 \\
\hline & $\%$ & $63,33 \%$ & $26,67 \%$ & $0,00 \%$ & $10,00 \%$ & $82,76 \%$ \\
\hline \multirow{2}{*}{ Masculino } & $\mathrm{n}$ & 13 & 14 & 1 & 6 & 28 \\
\hline & $\%$ & $38,24 \%$ & $41,18 \%$ & $2,94 \%$ & $17,65 \%$ & $77,78 \%$ \\
\hline \multirow{2}{*}{ Total } & $\mathrm{n}$ & 32 & 22 & 1 & 9 & 52 \\
\hline & $\%$ & $50,00 \%$ & $34,38 \%$ & $1,56 \%$ & $14,06 \%$ & $80,00 \%$ \\
\hline
\end{tabular}

\begin{tabular}{|c|c|c|c|c|c|c|}
\hline \multicolumn{6}{|c|}{ Tabla 6. Valoración de la utilización de pulsímetros para entrenar $(\mathrm{n}=61)$. Imprescindible $(\mathrm{n}=59)$} \\
\hline Género & Casos & Bien & Regular & Mal & No hay & Impres. \\
\hline \multirow{2}{*}{ Femenino } & $\mathrm{n}$ & 3 & 3 & 0 & 23 & 8 \\
\cline { 2 - 7 } & $\%$ & $10,34 \%$ & $10,34 \%$ & $0,00 \%$ & $79,31 \%$ & $29,63 \%$ \\
\hline \multirow{2}{*}{ Masculino } & $\mathrm{n}$ & 3 & 3 & 4 & 22 & 13 \\
\cline { 2 - 7 } & $\%$ & $9,38 \%$ & $9,38 \%$ & $12,50 \%$ & $68,75 \%$ & $40,63 \%$ \\
\hline \multirow{2}{*}{ Total } & $\mathrm{n}$ & 6 & 6 & 4 & 45 & 21 \\
\cline { 2 - 7 } & $\%$ & $9,84 \%$ & $9,84 \%$ & $6,56 \%$ & $73,77 \%$ & $35,59 \%$ \\
\hline \multirow{2}{*}{ Leyenda: (Bien) valoración de bien, (Regullar) valor ación de regular, (Mal) valoración de mal, (No hay) no tengo } \\
entrenador, (Impres.) es imprescindible para los entrenamientos.
\end{tabular}

De manera general, el $50,00 \%$ manifiesta que los materiales con los que entrena en su instalación habitual son buenos, mientras que el $35,94 \%$ manifiesta que son regulares o malos. Desgraciadamente también nos encontramos con un $14,06 \%$ de deportistas que no poseen material específico en sus entrenamientos.

Uno de los medios básicos y habituales para controlar y evaluar los entrenamientos de los deportistas en la actualidad, en las diferentes disciplinas que nos encontramos son los pulsímetros. Sin embargo, en esta disciplina observamos que el $73,77 \%$ de los deportistas manifiestan no entrenar con ellos y, por otro lado, tampoco manifiesta un alto porcentaje en cuanto a su relevancia en el entrenamiento; manifestando sólo el 35,59\% que son imprescindibles para su deporte.

\subsection{En relación al entrenamiento}

El mayor porcentaje de deportistas que entrenan salvamento acuático deportivo entrena en grupo $(93,15 \%)$. Aunque este deporte se caracteriza por realizar pruebas de carácter individual, también contempla pruebas de equipos. Además, el sistema de competición propicia la participación por equipos, donde cada uno de los componentes en sus respectivas pruebas y en sus respectivos equipos, suman puntos para el colectivo. El nivel que poseen los compañeros de entrenamiento, es en el $67,69 \%$ de los casos similar al del deportista, mientras que el $26,15 \%$ manifiesta entrenar con compañeros que poseen un mayor nivel que ellos.

La media de años dedicados a este deporte como deportista en competición es ligeramente superior a los cinco años $(5,29 \pm 2,92)$. Ahora bien, las chicas promedian unos datos superiores en cuanto a los meses de entrenamiento al año, sobrepasando los 10 meses que obtienen como dato medio.

Además, el número de días que los deportistas entrenan a la semana se establece en 4,03 $\pm 1,69$ como término medio, lo que nos indica que prácticamente todos los deportistas entrenan una vez al día. Cabe destacar que en cada día de entrenamiento se realiza una sesión de hora y media de duración.

\begin{tabular}{|c|c|c|c|c|c|c|c|}
\hline \multirow{2}{*}{ Género } & \multicolumn{5}{|c|}{ Tabla 7. Tipo de trabajo de fuerza específica ( } & \multicolumn{2}{|c|}{ Trabajo en agua } \\
\hline & Casos & Pesas & Gomas & Otros & Palas & \begin{tabular}{|l|} 
Resistencia \\
\end{tabular} & Otras \\
\hline \multirow{2}{*}{ Femenino } & $\mathrm{n}$ & 15 & 14 & 4 & 17 & 6 & 0 \\
\hline & $\%$ & $45,45 \%$ & $42,42 \%$ & $12,12 \%$ & $73,91 \%$ & $26,09 \%$ & $0,00 \%$ \\
\hline \multirow{2}{*}{ Masculino } & $\mathrm{n}$ & 19 & 14 & 3 & 16 & 8 & 1 \\
\hline & $\%$ & $52,78 \%$ & $38,89 \%$ & $8,33 \%$ & $64,00 \%$ & $32,00 \%$ & $4,00 \%$ \\
\hline \multirow{2}{*}{ Total } & $\mathrm{n}$ & 34 & 28 & 7 & 33 & 14 & 1 \\
\hline & $\%$ & $49,28 \%$ & $40,58 \%$ & $10,14 \%$ & $68,75 \%$ & $29,17 \%$ & $2,08 \%$ \\
\hline \multicolumn{8}{|c|}{ 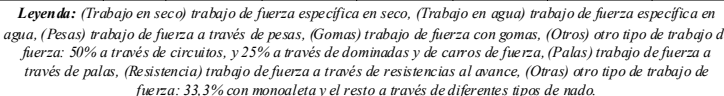 } \\
\hline
\end{tabular}

El trabajo de fuerza específica es realizado por un $53,42 \%$ de los deportistas, siendo superior este trabajo en mujeres que en varones. $\mathrm{Si}$ bien, este trabajo se distribuye en dos grandes bloques; por un lado el trabajo de fuerza en seco y, por otro, el trabajo específico de fuerza en el agua. De entre ellos, el más realizado es el trabajo en seco, aunque los porcentajes son muy similares.

Analizando más profundamente este trabajo de fuerza específico, nos encontramos con las diferentes formas de trabajar esta capacidad física, tanto en seco como en agua (Tabla 7). Así nos encontramos que en el medio terrestre este tipo de trabajo se realiza a través de un trabajo de pesas y de gomas, obteniendo resultados similares, aunque ligeramente superiores, en el trabajo de pesas en los varones. Sin embargo, en el medio acuático, el trabajo de fuerza se realiza mayoritariamente a través de las palas (68,75\%), seguido de otros métodos de trabajo que se basan en resistencias al avance en el agua $(29,17 \%)$.

En relación al tipo de entrenamiento, podemos apreciar que los mayores porcentajes realizados en entrenamientos son de tipo táctico y estratégico respectivamente (Tabla 8). Estos datos son comprensibles al entender el sistema de puntuación que se realiza en las diferentes competiciones. En ellas, el deportista puede ser el ganador o perdedor, pero en función de su puesto otorgará unos puntos a su club, equipo o selección. Por tanto, aparecen planteamientos, aunque el carácter de las pruebas sea individual, de ayuda a compañeros del mismo equipo, para que queden en mejor posición que los rivales, aunque no se gane la prueba.

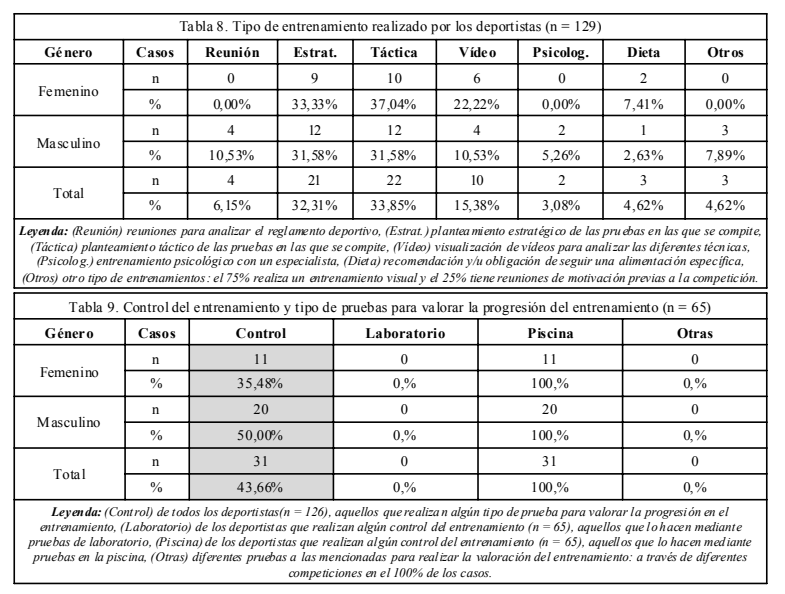

En los entrenamientos que siguen se reflejan principalmente en visionado de videos de diferentes competiciones y deportistas $(15,38 \%)$, para analizar aspectos técnicos y de competición, además de reuniones previas a la competición $(6,15 \%)$ para analizar y recordar los aspectos reglamentarios de las pruebas.

En menor porcentaje los deportistas realizan entrenamiento psicológico $(3,08 \%)$ y/o alimenticio $(4,62 \%)$, que creemos deberían estar más presentes en deportistas de alta competición, como son los aquí estudiados.

Uno de los datos que nos encontramos en este estudio es que el $61,64 \%$ de los deportistas practica sólo salvamento acuático. Estos datos no se corresponden para nada con el estudio de Abraldes (2006), donde manifiesta que a nivel nacional, el 65,63\% de los deportistas simultanea con otros deportes en la misma temporada. Este dato además está relacionado con el hecho de que el 75,90\% de esos deportistas le dedican más tiempo al otro deporte con respecto al salvamento Acuático. En nuestro estudio, estos datos también se vuelven contradictorios, ya que observamos que los deportistas de la comunidad gallega, le dedican más tiempo al salvamento acuático $(75,00 \%)$, en detrimento del número de deportistas que entrenan con más asiduidad el otro deporte $(25,00 \%)$.

De entre los diferentes deportes practicados por los deportistas de salvamento acuático deportivo hay que destacar que el $14,29 \%$ practica el fútbol, seguido por la práctica de la natación y el baloncesto $(8,57 \%)$. 
Estos datos tampoco concuerdan con los encontrados en el estudio de Abraldes (2006) donde la mayoría de los deportistas practicaba natación $(61,5 \%)$, ya que es el deporte más similar en cuanto a los aspectos técnicos y el medio en el que se desarrolla y, por tanto, el que mejores y más transferencias positivas presenta.

Otro dato característico de cara a evaluar la evolución de los entrenamientos son las pruebas, test o métodos de control que determinan o indican la evolución del mismo. En este caso, nos encontramos con un porcentaje del $43,66 \%$ que realizan este tipo de pruebas o test de valoración. Donde las pruebas se realizan en todos los casos a través de controles en la piscina, mientras que el laboratorio (análisis de lactato, volumen máximo de oxígeno, test de esfuerzo, etc.) u otros sistemas de control no se realizan.

\section{Conclusiones}

Las conclusiones encontradas en este estudio se pueden reflejar de la siguiente forma:

- La formación de los entrenadores es aceptable, aunque no ideal, pues mayoritariamente está más orientada al mundo de la natación que al salvamento acuático deportivo.

- Un alto porcentajes de los deportistas $(87,69 \%)$ valora como buena la piscina en la que entrena, siendo imprescindible para el entrenamiento en el $94,37 \%$ de los deportistas.

- El gimnasio es regular o malo en el 37,88\% de los casos, y sin embargo lo consideran imprescindible para un buen entrenamiento el $70,15 \%$ de los deportistas.

- El material específico es valorado como regular o malo en el $35,94 \%$ de los casos, mientras que el $14,06 \%$ de los deportistas no dispone de él para su entrenamiento.

- Los pulsímetros sólo son utilizados por el 26,23\% de los deportistas, ya que el $73,77 \%$ manifiesta que no los tiene en sus entrenamientos. Al mismo tiempo no los consideran imprescindibles para su trabajo.

- Los tipos de entrenamiento más practicados son del tipo táctico $(33,85 \%)$ y estratégico $(32,31 \%)$ siendo de menor porcentaje otros como el entrenamiento de visualización de vídeos, psicológico, reuniones técnicas o control alimentario.

Se realiza un poco menos de una sesión de entrenamiento diaria a la semana, con una duración total de hora y media, durante 10 meses al año.

El trabajo de fuerza específica es realizado por un $53,42 \%$ de los deportistas, tanto en seco como en agua. Aquí, el trabajo de fuerza se realiza mayoritariamente a través de las palas $(68,75 \%)$, seguido de otros métodos de trabajo que se basan en resistencias al avance en el agua $(29,17 \%)$

- El 61,64\% de los deportistas practica sólo salvamento acuático y de los que simultanean su práctica con otra actividad deportiva, éstos le dedican más tiempo al salvamento acuático $(75,00 \%)$, en detrimento del número de deportistas que entrenan con más asiduidad el otro deporte $(25,00 \%)$.

- Sólo el 43,66\% de los deportistas realizan un control del entrenamiento mediante pruebas o test de valoración, y en el 100\% de los casos se hace con pruebas en la piscina.

\section{Bibliografía}

Abraldes, J. A. (2002): Salvamento Acuático y Deporte: un estudio de los recursos humanos en las playas de Galicia, intervenciones en los rescates y su relación con el ámbito deportivo. ACoruña: Xaniño Editorial. Tesis doctoral. Formato digital.

Abraldes, J. A. (2004): Salvamento Acuático Deportivo. Un deporte solidario. ACoruña: Xaniño Editorial.

Abraldes, J.A. (2005). La formación de los entrenadores responsables de los deportistas que participan en el campeonato de España de Salvamento Acuático Deportivo del año 2004. $4^{\circ}$ Congreso de Salvamento y Socorrismo de Galicia. ACoruña.

Abraldes, J. A. (2005): Estudio de los factores que influyen en el proceso de formación deportiva en salvamento y socorrismo. International Lifesaving Congreso (pp. 1-20). Alicante.

Abraldes, J. A. (2006). Aspectos relevantes de los entrenamientos en deportistas de alto nivel en salvamento acuático deportivo. $I V$ Congreso de la Asociación Española de Ciencias del Deporte. La Coruña.

Abraldes, J. A., Barroso, A., Rodríguez, N., Soares, S., Pinto, R. J. y Vilas-Boas, J. P. (2006). Salvamento Acuático Deportivo. Pruebas de piscina. A Coruña: Federación de Salvamento e Socorrismo de Galicia. [Formato DVD].

Federación de Salvamento e Socorrismo de Galicia. (1999). Primer congreso de Salvamento y Socorrismo de Galicia. Salvamento y Socorrismo: Una aproximación interdisciplinar. ACoruña: ICongreso de Salvamento y Socorrismo de Galicia.

Federación de Salvamento e Socorrismo de Galicia. (2001). $2^{\circ}$ Congreso de Salvamento y Socorrismo de Galicia. ACoruña: Diputación provincial de ACoruña.

Fernández Balboa, J. M. (1997). La investigación en la Educación Física española: un índice para el futuro. Apunts. Educación fisica y deportes, 50, 100-106.

Gómez, J. (1990). Metodología de encuesta por muestreo. En Arnau, J., Anguera, M. T. y Gómez, J. Metodología de la investigación en ciencias del comportamiento. (pp. 237-310). Murcia: Universidad de Murcia.

Palacios, J., Iglesias, O., Zanfaño, J., Angueira, G. y Parada, E. (1998). Salvamento Acuático, Salvamento Deportivo y Psicología. A Coruña: Xaniño Editorial.

Pascual, L. F. (2004). Salvamento Acuático Profesional en piscinas y su relación con el salvamento acuático deportivo en aguas cerradas. Universidad de Zaragoza. Zaragoza.

\section{Agradecimientos}

Quisiera expresar mi agradecimiento a la Federación de Salvamento y Socorrismo de Galicia (FESSGA) así como a todos los deportistas que participaron en el estudio, por su apoyo y paciencia en la presente investigación. Por otro lado, agradecer a la Facultad de Ciencias de la Actividad Física y el Deporte de la Universidad Católica San Antonio de Murcia (UCAM), por permitir el desarrollo de esta investigación. 